\title{
Support diagnostique en cancérologie : techniques complémentaires et apport de la télépathologie, exemple de l'hématopathologie
}

\author{
Diagnostic aids in cancer medicine: additional methods and the role of telepathology \\ in haematological disease
}

\author{
M. Raphaël · N. Hurwitz \\ C Springer-Verlag France 2011
}

La précision du diagnostic histologique ou cytologique est le prérequis indispensable à la prise en charge adéquate d'un patient atteint d'une pathologie tumorale.

La morphologie, gold standard de toute analyse initiale, est désormais complétée par une approche multidisciplinaire : immunophénotypage, cytogénétique, biologie moléculaire et génomique. Les récentes classifications de l'OMS, en l'occurrence celle des hémopathies malignes [1], identifient des catégories tumorales reposant sur des critères morphologiques, immunophénotypiques, cytogénétiques et moléculaires caractérisant selon les aspects cliniques des entités à part entière. Celles-ci justifient une attitude thérapeutique bien définie et ciblée. L'identification de marqueurs spécifiques devient ainsi indispensable pour le traitement et le suivi d'une pathologie maligne.

Cette approche diagnostique sous-entend des techniques histologiques standard constantes et de bonne qualité complétées par des abords biologiques nécessitant un équipement performant et un personnel entraîné. L'ensemble de ces conditions étant rarement mis en place dans les pays émergents, cela aggrave les inégalités d'accession aux soins constatées dans ces pays.

La télépathologie apporte des solutions aux difficultés de communications liées à l'isolement, au faible nombre de pathologistes et aux longues distances séparant plusieurs centres de pathologie ou hôpitaux universitaires munis de service d'anatomie et cytologie pathologiques. Toutefois,

M. Raphaël $(\bowtie)$

Service d'hématologie et immunologie biologiques, cytogénétique, AP-HP, CHU Bicêtre, université Paris-Sud, CNRS UMR8126, Alliance mondiale contre le cancer (AMCC), International Network for Cancer Research and Treatment (INCTR), le Kremlin Bicêtre, F-94275 cedex, France

e-mail : martine.raphael@bct.aphp.fr

N. Hurwitz

Département de pathologie, université de Bâle,

Pathology Education Program, INCTR, Suisse l'optimisation de cet outil de communication nécessite le renforcement des techniques standard avec l'amélioration de l'équipement et la formation des techniciens. Ces préalables sont indispensables au développement de la télépathologie. Seules les images de bonne qualité résultant d'une technique correcte sont interprétables et permettent des échanges entre pathologistes. L'identification des facteurs limitants et des besoins spécifiques des différents pays d'Afrique est nécessaire avant toute action de soutien diagnostique.

La réactivité et la rapidité des échanges en utilisant les moyens via internet sont un apport considérable pour l'amélioration des techniques standard et la mise en place des techniques d'immunohistochimiques nécessaires à la caractérisation des hémopathies malignes, en particulier lymphoïdes. En effet, à l'ère des thérapies ciblées, et devant les progrès thérapeutiques liés aux anticorps monoclonaux et plus particulièrement, dans la pathologie lymphoïde avec le rituximab (anti-CD20), il est indispensable d'identifier les lymphomes non hodgkiniens de nature $\mathrm{B}$ pouvant bénéficier d'un tel traitement.

Des expériences de télépathologie et de transmission d'images cytologiques pouvant apporter une aide au diagnostic ont déjà été menées en hématologie il y a plusieurs années [2]. De telles expériences ont aussi été développées dans plusieurs pays confrontés à l'isolement des pathologistes [3]. Cet isolement peut être rompu par l'organisation de réseaux afin d'atteindre les centres les plus éloignés, de faciliter le diagnostic et d'assurer la formation.

La convivialité du logiciel utilisé est une des clés pour la réussite d'une mise en réseau. Parmi des expériences déjà menées, l'exemple de l'hématopathologie utilisant le logiciel iPath développé par l'université de Bâle est une application Web fonctionnelle depuis 2001 [4]. Celle-ci, déjà utilisée depuis plusieurs années dans différents pays émergents, démontre la fonctionnalité de ce type de connexion entre un panel de pathologistes experts et des pathologistes ou hématologistes soumettant des cas pour consultation $[5,6]$. 
Ce même logiciel permet de collecter les données cliniques, biologiques et d'imagerie permettant des analyses clinicobiologiques dans le cadre d'une recherche clinique.

L'utilisation de la transmission d'images via internet est très utile pour les relectures des lames de biopsies ou de frottis de patients inclus dans des protocoles thérapeutiques. De telles expériences ont déjà été développées pour la relecture de lames de frottis sanguins et médullaires dans le cadre de protocoles multicentriques de groupes coopérateurs de traitement en France [7]. Cette démarche de relecture est en cours de mise en place dans les pays d'Afrique francophone comme en Afrique anglophone. Ces relectures via la télépathologie sont connectées aux protocoles de traitement, en particulier ceux des lymphomes de l'enfant, conduits sous l'égide du GFAOP (Groupe franco-africain d'oncologie pédiatrique) dans les pays francophones [8] ou de l'INCTR (International Network for Cancer Traitment and Research) dans les pays anglophones [9]. Ces relectures quasi immédiates des cas inclus aboutiront à un pourcentage élevé de seconde lecture cytologique ou histologique et à l'identification de cas difficiles pour lesquels des techniques complémentaires d'immunohistochimie, de cytogénétique ou de biologie moléculaires sont nécessaires.

L'enseignement $[6,10]$ bénéficie aussi de cette technologie de transmission. Plusieurs expériences ont déjà été menées ainsi, les échanges rapides entre plusieurs laboratoires de pathologie permettent une aide à la formation à la fois pratique pour les techniques d'immunohistochimie mais aussi théorique par l'analyse des mêmes territoires pathologiques. Le logiciel iPath est aussi très utile pour la formation avec la possibilité de créer un groupe d'experts en parallèle avec un groupe de discussions diagnostiques [5]. Cet apprentissage est réalisé via Internet où l'enseignant et le tuteur peuvent commenter la même lame à distance via les nouveaux moyens de téléphonie et de vidéotransmission.

L'ensemble de cette approche aboutit à une amélioration des soins donnés aux patients par l'obtention de diagnostics rapides et fiables, à un enseignement interactif pour les étudiants en médecine et à la formation médicale continue pour les médecins spécialistes.

La télépathologie s'intègre dans une démarche plus générale de télémédecine impliquant les pays avec leurs organismes de tutelle ainsi que les institutions académiques [11]. Un respect mutuel et un climat de confiance sont indispensables à la création de tels réseaux synergiques.

We speak about the loneliness of a long distance runner, but there may be no one more lonelier than a surgical pathologist working solo... easy access to consultation is a prerequisite for accurate diagnosis and accordingly for optimal patient care.

Stephen Sternberg, Surgical pathology Preface to the first edition 1989

\section{Références}

1. Swerdlow SH, Campo E, Harris NL, et al (eds) (2008) WHO classification of tumors of hematopoietic and lymphoid tissues, 4th edition. IARC Press (Lyon)

2. Flandrin G (2004) La télépathologie en hématologie. Rev Fr Lab 364:55-60

3. Baruah MK (2005) The practice of telepathology in India. J Postgrad Med 51:316-8

4. Brauchli K, Helfrich M, Christen H, et al (2002) The future of telepathology. An internet "distributed system" with "open standards". Pathologie 23:198-206

5. Brauchli K, Oberli H, Hurwitz N, et al (2004) Diagnostic telepathology: long-term experience of a single institution. Virchows Arch 444:403-9

6. Ilunga Nikulu J, Kyabu Kabila V, Mwenze Mwadi, et al (2011) La télépathologie : 1 an d'expérience franco-lushoise : une réalité. $\mathrm{XI}^{\mathrm{es}}$ Journées franco-africaines de pathologie, la division d'Afrique francophone de l'Académie internationale de pathologie (DAF-AIP), 30/01-02/02 2011, Lomé, Togo

7. Leymarie V, Flandrin G, Noguera ME, et al (2006) Telehematology: a pilot experience of cytological diagnosis of acute myeloid leukemia via the Internet. A GOELAMS study. Haematologica 91:1285-6

8. Traoré F, Coze C, Atterby JJ, et al (2010) Cyclophosphamide monotherapy in children with Burkitt lymphoma: a studyfrom the French African Pediatric Oncology Group (GFAOP). Pediatr Blood Cancer on line

9. Magrath IT (2006) Treatment of Burkitt lymphoma in children and adults: lessons from Africa. Curr Hematol Malig Rep $1: 230-40$

10. Giansanti D, Castrichella L, Giovagnoli MR (2008) Telepathology training in a master of cytology degree course. J Telemed Telecare 14:338-41

11. Williams S, Henricks WH, Becich MJ, et al (2010) Telepathology for patient care: what am i getting myself into? Adv Anat Pathol $17: 130-49$ 